\title{
Piston Position Sensing and Control in a Linear Compressor using a Search Coil
}

\author{
Kun Liang*, Richard Stone, Mike Dadd, Paul Bailey \\ Department of Engineering Science, University of Oxford, Oxford, UK, OX1 3PJ \\ ${ }^{*}$ Corresponding author: Department of Engineering Science, University of Oxford, Oxford \\ OX1 3PJ, UK. Email address: kun.liang@eng.ox.ac.uk (K. Liang)
}

\begin{abstract}
Linear compressors with clearance seals are well established for cryocoolers using either a Stirling cycle or pulse tube. However, both piston stroke and piston offset require detection and control in valved compressors. In order to manufacture linear compressor in large scale, then a simple, robust, and cheap control system is required with a minimum of sensors and actuators. This work introduces an approach to sensing the position of the piston using a search coil. The piston stroke is detected by calculating the peak-to-peak flux linkage in relation to the measured drive voltage and current. The piston offset is proportional to an integrated modulus of the back electromotive force (emf) of the search coil. Two PID (proportional-integral-derivative) controllers were developed to control the piston stroke by adjusting the drive voltage and a solenoid valve and bleed flow to control the piston offset. Experimental results validate the proposed compressor control technique.
\end{abstract}

Keywords: Linear compressor, control system, search coil, piston stroke, piston offset 
NOMENCLATURE

$\begin{array}{ll}\text { A } & \text { area }\left(\mathrm{mm}^{2}\right) \\ C & \text { constant } \\ \text { COP } & \text { coefficient of performance } \\ f & \text { frequency }(\mathrm{Hz}) \\ I & \text { current }(\mathrm{A}) \\ k & \text { factor of displacement or spring rate }(\mathrm{N} / \mathrm{mm}) \\ \text { LVDT } & \text { linear variable differential transformer } \\ M & \text { mutual inductance }(\mathrm{H}) \\ P & \text { pressure }(\text { bar) or power }(\mathrm{W}) \\ \text { PID } & \text { proportional-integral-derivative } \\ \text { PWM } & \text { pulse-width-modulation } \\ R & \text { resistance }(\Omega) \\ \text { RMS } & \text { root mean square } \\ S & \text { stroke (mm) } \\ V & \text { voltage }(\mathrm{V}) \\ X & \text { displacement }(\mathrm{mm})\end{array}$

\section{Subscripts}

flux linkage (Weber)

\section{Subscripts}

$\begin{array}{ll}0 & \text { initial } \\ 1 & \text { cylinder } \\ \mathrm{a} & \text { amplitude } \\ \mathrm{b} & \text { body } \\ \text { dis } & \text { discharge } \\ \text { emf } & \text { electromotive force } \\ \mathrm{g} & \text { gas } \\ \text { int } & \text { integration } \\ \mathrm{m} & \text { mean or main or mechanical } \\ \mathrm{s} & \text { search oil } \\ \text { suc } & \text { suction }\end{array}$




\section{Introduction}

A linear compressor is an attractive proposition for electronics cooling applications because it offers several benefits compared to traditional compressor technologies, as pointed out by Bailey et al. (2011). A linear compressor does not have a crank mechanism to drive the piston but is driven directly by a linear motor. Oil-free operation is possible, and this is a significant advantage with respect to the heat transfer performance of the condenser and the evaporator in a refrigeration system as it would allow the use of compact heat exchangers. The absence of oil widens both the choice of refrigerants and their operating temperature range. Eliminating the need for the oil return reduces constraints on pipe sizing and will lead to a reduction in pressure drop losses. In a high efficiency linear machine, the piston operates resonantly in order to minimise the drive current and ohmic losses. Resonance is determined by the moving mass and stiffness, which has at least two components, the mechanical springs and the gas spring. Bradshaw et al. (2011) concluded that the potential for this technology to be scaled to small physical sizes is better than for conventional compressors.

Sunpower have been developing linear technologies for a long period. LG have licensed Sunpower's linear technology and have been marketing linear compressor systems for household refrigeration since 2002. A more recent development by Embraco is an oil-free linear compressor designed for R134a and R600a. Embraco launched their oil-free linear compressor in 2014 with a license from Fisher \& Paykel. Bradshaw (2012) has also built a linear compressor model that was validated by testing a prototype linear compressor that used a moving magnet motor.

In a linear compressor, the flow rate is controlled by amplitude modulation which means that there is no need for stop-start operation in which temperature gradients have to be re-established; Tassou et al. (1983). The linear compressor is a free piston system, so that the cooling capacity can be modulated by the piston stroke control. The stroke is controlled by adjusting the drive voltage (excitation voltage). Huang and Chen (2002) found that the stroke of the piston in a linear compressor varies markedly with the outlet pressure variation. A slight increase in outlet pressure will cause a dramatic decrease in stroke. Huang and Chen studied the system dynamics of a linear compressor and designed a controller to regulate both the stroke and frequency of the compressor during operation using a displacement transducer. Kim and Jeong (2013) and Kim et al. (2011) proposed an inherent capacity modulation technique for the LG linear compressor by developing a numerical model of the compressor system. The capacity modulation is based on the clearance volume which is the distance from the top position of the piston to the cylinder head.

Fig. 1 shows the schematic of a free-piston linear compressor system. Apart from stroke control, the linear compressor requires control of the piston offset. The problem of piston offset (or drift) in a linear compressor is caused by the differential pressure generated across a radial clearance seal which has a fluctuating pressure on one side of it (piston-cylinder) and an almost constant pressure on the other (body). The pressure differential is given by

$$
\Delta P=P_{1, \mathrm{~m}}+P_{1, \mathrm{a}} \sin (\omega t)-P_{\mathrm{b}}
$$

where $P_{\mathrm{b}}$ is the pressure in the motor body and $P_{1, \mathrm{~m}}$ is the mean pressure in the cylinder. 


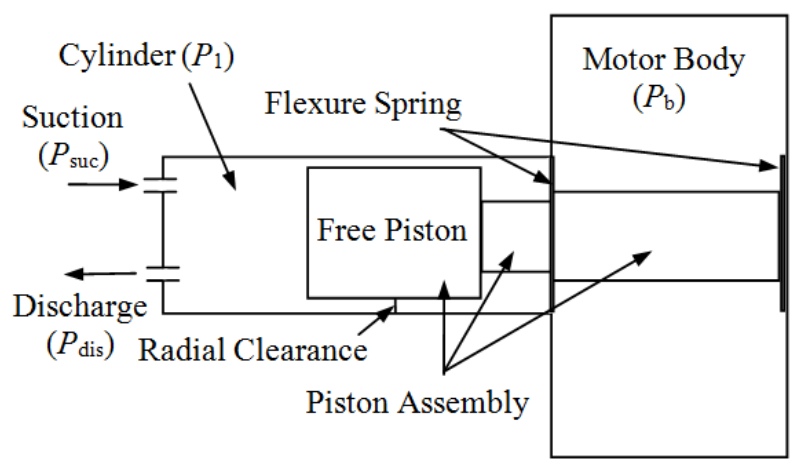

Fig. 1 Schematic of the linear compressor system

If the body pressure $P_{\mathrm{b}}$ is equal to the mean working pressure $P_{1, \mathrm{~m}}$, then the net volumetric flow taken round one cycle is zero. The mass flow rate, however, is proportional to the mean density, and this is higher when the piston is closer to cylinder head than when it is closer to compressor body side. Thus there will be a net mass flow from the working side to the body volume across the clearance seal, which will decrease the mean working pressure and increase the body pressure. Eventually an equilibrium point will be reached when this effect is counterbalanced by the pressure differential in the opposite direction.

If there is a difference between the mean working pressure $P_{1, \mathrm{~m}}$ and the body pressure $P_{\mathrm{b}}$ there will be a net axial force, which is only counteracted by the mechanical springs, and this will result in a shift of the mean position of the piston. Note that if the piston is not oscillating about the 'mechanical zero' of the springs, there will be a reduction in the useful stroke of the compressor. If there is no control and if the piston offset is large, then the piston may hit the cylinder head.

Bradshaw (2012) also reported piston drift from its datum position during operation, and the linear compressor model showed that the drift decreased with the dead (clearance) volume and increased with the stroke-diameter ratio of the piston. Spoor and Corey (2004) derived simple expressions for the time-average mass flux through a clearance seal, and predicted the piston offset by assuming that the piston was acting against enclosed volumes on either side. Zou et al. (2012) employed a formula (from Choe and Kim, 2000) to calculate the piston offset and validated this method by experiments.

Kim et al. (2009) experimentally concluded that it is very important to link the operating frequency with the natural frequency to maximise the linear compressor efficiency. Lin et al. (2007) proposed a novel resonant frequency tracking technique in order to achieve high efficiency operation of a linear compressor system. By evaluation of the input power to the compressor and implementing a 'perturb and observe' algorithm, the frequency of the motor current can be adjusted to match the resonant frequency of the compressor.

The design and performance of the oil-free linear compressor studied here has been reported already in Liang et al. (2014) and Liang et al. (2012). This work focuses on the control of the linear compressor. The piston stroke will be derived from the peak-to-peak flux linkage in a search coil and the piston offset will be detected by the flux linkage difference. No position transducer will be needed. This will be beneficial to the large scale manufacture for the next stage of development. Control of the stroke is based on the drive voltage while the piston offset is controlled by adjusting the bleed flow rate from the compressor to the body side. The resonant frequency will be modelled, so the linear compressor can be operated at resonance for each operating point. The control system for stroke, 
offset and frequency have been implemented in LabVIEW to demonstrate the feasibility and efficiency.

\section{Linear Compressor Prototype}

Fig. 2 (a) and (b) show the key components of the moving magnet linear compressor. The linear suspension system has two sets of spiral springs to maintain the alignment of the piston within the cylinder. The accuracy of this linear system permits a radial clearance between the piston and cylinder of about $10 \mu \mathrm{m}$ to be used. This is sufficient to avoid wear, while keeping leakage losses to a minimum. The static assembly has 4 magnetic circuits consisting of coils wound on laminated and slotted cores to form an air gap. The moving assembly (3 rectangular magnets in a line within the air gap) is attached to the piston. When an alternating current is supplied to the cores, an alternating axial force is induced in the moving magnet assembly, allowing the piston to reciprocate at the same frequency. Further details of the compressor design have been reported by Bailey et al. (2009). Table 1 gives the design specification of the moving magnet compressor, which uses two identical compressor halves mounted back-to-back and in-line to minimise exported vibrations. An adiabatic efficiency of $42 \%-60 \%$ and a motor efficiency of $71 \%$ - $89 \%$ were maintained over a fairly wide range of operating parameters, suggesting that systems using this type of compressor could have good part load efficiency. A Coefficient of Performance (COP) of 3.2 was achieved at a cooling capacity of $384 \mathrm{~W}$ with an evaporator temperature of $20^{\circ} \mathrm{C}$ and a condenser temperature of $50^{\circ} \mathrm{C}$ using R134a.

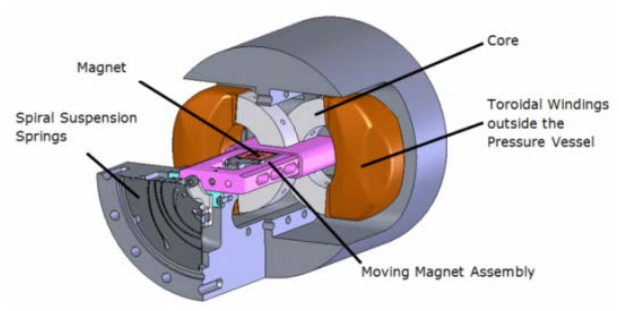

(a) Moving magnet motor design

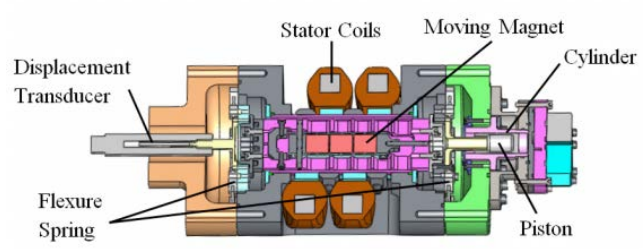

(b) Cross section of the linear compressor and motor

Fig. 2 Design of the 100W Oxford moving magnet compressor

Table 1 Specification of each linear compressor half

\begin{tabular}{ll}
\hline Total mass of magnet/piston $(\mathrm{kg})$ & 0.66 \\
Piston diameter $(\mathrm{mm})$ & 18.99 \\
Total series $^{1}$ resistance of coils $(\Omega)$ & 14 \\
Peak shaft force $(\mathrm{N})$ & 84 \\
Peak current at peak force $(\mathrm{A})$ & 1.29 \\
Flexure stiffness (Total) $\left(\mathrm{kN} \mathrm{m}^{-1}\right)$ & 17 \\
Maximum stroke (mm) & 14.00 \\
\hline
\end{tabular}

${ }^{1}$ The four motor coils were connected with two coils in series then each pair in parallel to give a resistance of $\sim 3.5 \Omega$ for each compressor half.

\section{Piston Position Sensing}




\subsection{Piston Stroke Detection}

In any linear electromagnetic transducer, the flux linkage $\Phi$ between coils and magnet systems varies with axial position $(x)$. For the condition where the coil current is zero, this can be expressed by:

$$
\Phi=f(x)
$$

where $x$ is a single valued function of $\Phi$.

If the flux linkage can be determined, then the piston/armature position can also be determined. This would allow the stroke and mean position also to be calculated.

The electromotive force (emf) generated in a coil through changes in flux linkage is given by:

$$
V_{\mathrm{emf}}=-\frac{d \Phi}{d t}
$$

However, the electromotive force $V_{\text {emf }}$ cannot be measured directly as the voltage across the coil generally comprises of additional terms.

Search coils are additional coils wound around the main drive coils that are used to detect changes in flux linkage through the voltage generated in them. They do not carry any current and hence they can be made of small diameter wire.

For a search coil, the voltage across it $V_{\mathrm{S}}$ can be written as

$$
\begin{aligned}
& V_{\mathrm{s}}=V_{\mathrm{emf}}+M \frac{d I}{d t} \\
& V_{\mathrm{s}}=-\frac{d \Phi}{d t}+M \frac{d I}{d t}
\end{aligned}
$$

where $\frac{d \Phi}{d t}$ is the change in flux linkage in the search coil generated by the piston/armature movement and $M \frac{d I}{d t}$ is the inductive voltage in the search coil generated by a change of current in the drive coil. $M$ is the mutual inductance between drive and search coils.

Integration of $V_{\text {emf }}$ allows a value of a flux linkage $\Phi$ to be calculated. However, without a means of determining the integration constant $C_{\mathrm{int}}$, the resulting value cannot be used to infer the axial position:

$$
\Phi=\int V_{\mathrm{emf}} d t+C_{\mathrm{int}}
$$

Integration of $V_{\mathrm{emf}}$ can however be used to estimate the stroke, particularly if $\Phi$ is a reasonably linear function of $x$, as the integration constant disappears in the calculation:

$$
\begin{gathered}
x=\Phi / k \\
S=\left(x_{\max }-x_{\min }\right)=\left(\Phi_{\max }-\Phi_{\min }\right) / k
\end{gathered}
$$

where $S$ is the piston stroke and $k$ is the factor of displacement.

Since the voltage across the coil $V_{\mathrm{s}}$ and drive current $I$ can be readily measured, the piston stroke can be determined if the mutual inductance can be given.

\subsection{Piston Offset Detection}


To sense the piston position, the integration constant $C_{\text {int }}$ in Equation (6) needs to be evaluated. A proximity sensor can be used to provide a pulse at a particular axial position $x_{0}$ for which the flux linkage $\Phi_{0}$ is known. This pulse is used to start the integration of $V_{\mathrm{emf}}$. As the initial values for $x$ and $\Phi$ are known, the integration constant can be determined.

When $t=0, x=x_{0}$, and $\Phi=\Phi_{0}=C_{\text {int }}$, then

$$
\Phi(t)=\int_{0}^{t} V_{\mathrm{emf}} d t+\Phi_{0}
$$

The calculated value of $\Phi(t)$ can then be used to infer the position $x(t)$ using Equation (7) or a lookup table. The required output from the proximity sensor is a pulse that corresponds with a particular axial position.

The piston offset $x_{\mathrm{m}}$ is the difference between the actual mean operating point of the piston and its datum position, where $x=0$. With the integration constant being calculated using a proximity sensor, the piston offset can be calculated.

Although it is possible to vary the offset for lower strokes so as to improve the volumetric efficiency, it has been found that maintaining the offset at zero leads to better performance because of the higher motor efficiency.

One advantage of maintaining a zero offset is that a full determination of $x(t)$ is not required and a simplified method can be used.

Linear motors generally have a symmetric flux linkage characteristic that can be expressed as:

$$
\text { For } x=0, \Phi=0, x>0, \Phi=f(x) \text { and for } x<0, \Phi=-f(|x|)
$$

It follows from this that if a proximity sensor is used to provide a pulse when the armature is at the datum position (midpoint of the motor) i.e. at $x=0$, then integrating the modulus of $V_{\mathrm{emf}}$ for two periods defined by $x>0$ and $x<0$ will result in identical values only if the offset is zero.

$$
\text { For } x=0, \Phi_{0}=C_{\mathrm{int}}=0, x<0, \Phi_{A}=\int_{0}^{t_{1}}\left|V_{\mathrm{emf}}\right| d t \text { and for } x>0, \Phi_{B}=\int_{t_{1}}^{t_{2}}\left|V_{\mathrm{emf}}\right| d t
$$

Note that 0 to $t_{1}$ is interval for $x<0, t_{1}$ to $t_{2}$ is interval for $x>0$.

It can be seen that if the mean value $x_{\mathrm{m}}$ is zero (no piston offset), then $\Delta \Phi=\Phi_{A}-\Phi_{B}=0$. However, when there is a piston offset $\left(x_{\mathrm{m}} \neq 0\right)$, the flux linkage difference $\Delta \Phi$ will not be zero. The calculated flux linkage difference $\Delta \Phi$ can therefore be used as the process variable to drive (for example) a PID controller, so as to maintain a zero offset. The advantage with this approach is that the target value for $\Delta \Phi$ is zero and an accurate relationship between position $x$ and flux linkage is not required.

Fig. 3 shows a diagram to illustrate calculating the flux linkage for two periods of displacement. The flux linkage $\Phi_{A}$ for period of $x>0$ is the area enclosed by the modulus of the search coil voltage $\left(\left|V_{\mathrm{emf}}\right|\right.$ and the pulse generated when the piston passes the datum position $(x=0)$. The actual piston displacement is plotted as well to give a piston offset of $-0.3 \mathrm{~mm}$. For demonstrating the sensing technique, a linear variable differential transducer (LVDT) was used to generate a pulse when $x=0$ as well as to measure the real-time piston displacement to validate the technique. Note that a negative displacement indicates that the piston has moved towards the cylinder head from the datum position. 


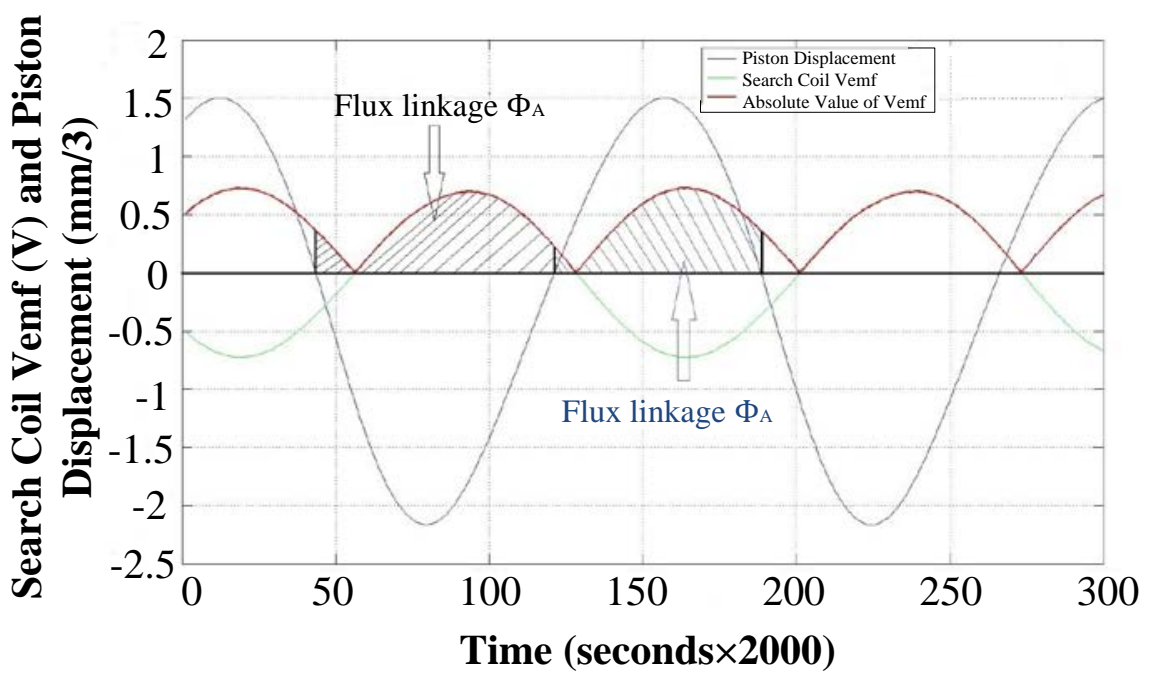

Fig. 3 Calculating flux linkage difference in the search coil to infer the piston offset

In reality, the pulse generated by a proximity sensor will not coincide exactly with the actual datum position of the linear compressor, indicating $\Phi_{A}-\Phi_{B}=\Phi_{R} \neq 0$. Nonetheless, providing the discrepancy is not very large, accurate centering of the piston can still be achieved if $\Phi_{A}-\Phi_{B}$ is replaced by $\Phi_{A}-\Phi_{B}-\Phi_{R}$ as the process variable.

\subsection{Search Coil}

The search coil (also known as an inductive sensor) is a sensor which measures the variation of the magnetic flux. A search coil with 10 turns was wound around the main coil (drive coil). Fig. 4 shows a search coil wound around a drive coil with a magnet sitting in the middle of the gap created by the slotted core. The terminals of the search coil were soldered to a capacitor $(0.033 \mu \mathrm{F})$ to reduce the noise in the measured inductive voltage.

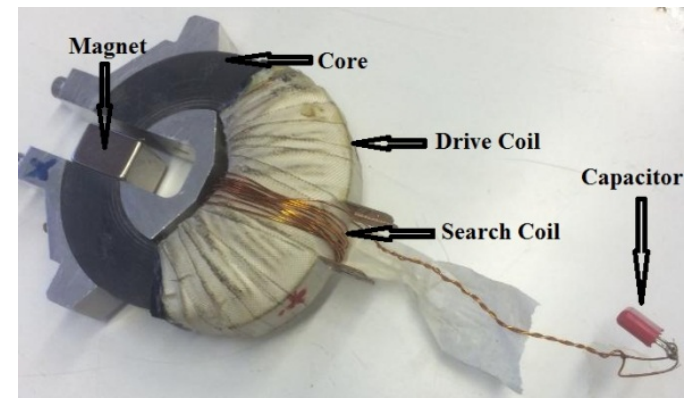

Fig. 4 Search coil wound around the drive coil (the magnet in the slot is for a static test)

As has been mentioned in Section 3.1, the mutual inductance between the search coil and drive coil needs to be defined so that the electromotive force $V_{\mathrm{emf}}$ can be determined using Equation (4).

A static test was conducted to measure the mutual inductance $M$. By arranging the search coil, drive coil, and magnet according to Fig. 4 and using an AC power source and a power meter, the mutual inductance can be measured at varying drive currents and frequencies. The schematic of the test is shown in Fig. 5. Without a magnet moving in the slot of the core, there is no changing flux linkage so 
that the inductive voltage from the measurement can be used to infer the mutual inductance. Note: all parameters were measured in terms of their RMS values.

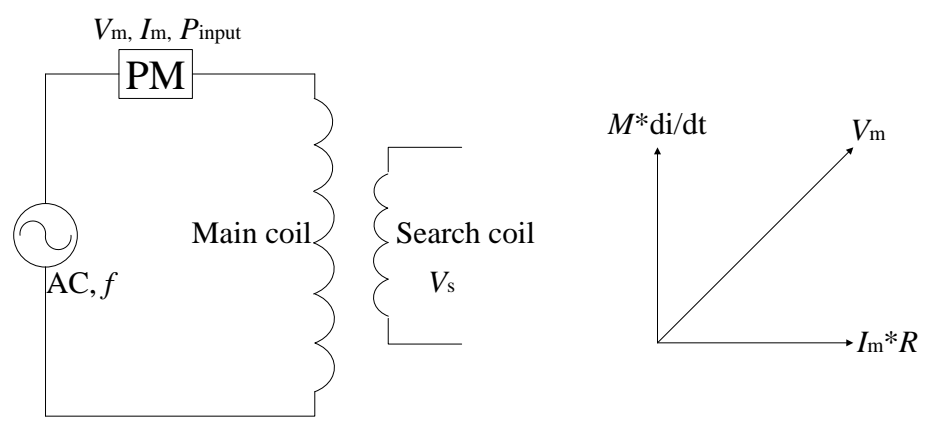

Fig. 5 Static test schematic of search coil (PM: power meter, $f$ : frequency, $m$ for main/drive coil and $s$ for search coil)

The operating frequency $f$ can be varied while the voltage $V_{\mathrm{m}}$, current $I_{\mathrm{m}}$ and power $P_{\text {input }}$ in the drive coil side can be measured using a power meter. The search coil voltage was measured by a digital voltmeter.

The inductance $L_{\mathrm{m}}$ and the resistance $R$ for the main/drive coil and mutual inductance $M$ can be inferred from equations as follow:

$$
\begin{gathered}
P_{\text {input }}=I_{\mathrm{m}}{ }^{2} * R \\
L_{\mathrm{m}} *(2 \pi f) * I_{\mathrm{m}}=\sqrt{V_{\mathrm{m}}^{2}-\left(I_{\mathrm{m}} * R\right)^{2}} \\
M *(2 \pi f) * I_{\mathrm{m}}=V_{\mathrm{s}}
\end{gathered}
$$

The calculated mutual inductances are plotted in Fig. 6. The mutual inductance $M$ is hardly changed with current and frequency, averaging at $1.5 \mathrm{mH}$ for currents ranging from $0.34 \mathrm{~A}$ to $1.3 \mathrm{~A}$. Without the magnet sitting in the middle, the maximum change in mutual inductance is only about $6.5 \%$. When a magnet is placed in the slot, the maximum change is about $10 \%$ at maximum current of $1.3 \mathrm{~A}$. The presence of the magnet offsets the flux so that the core saturated more quickly to give a lower mutual inductance. Note that $40 \mathrm{~Hz}$ and $1 \mathrm{~A}$ is the design condition for this prototype linear compressor. 


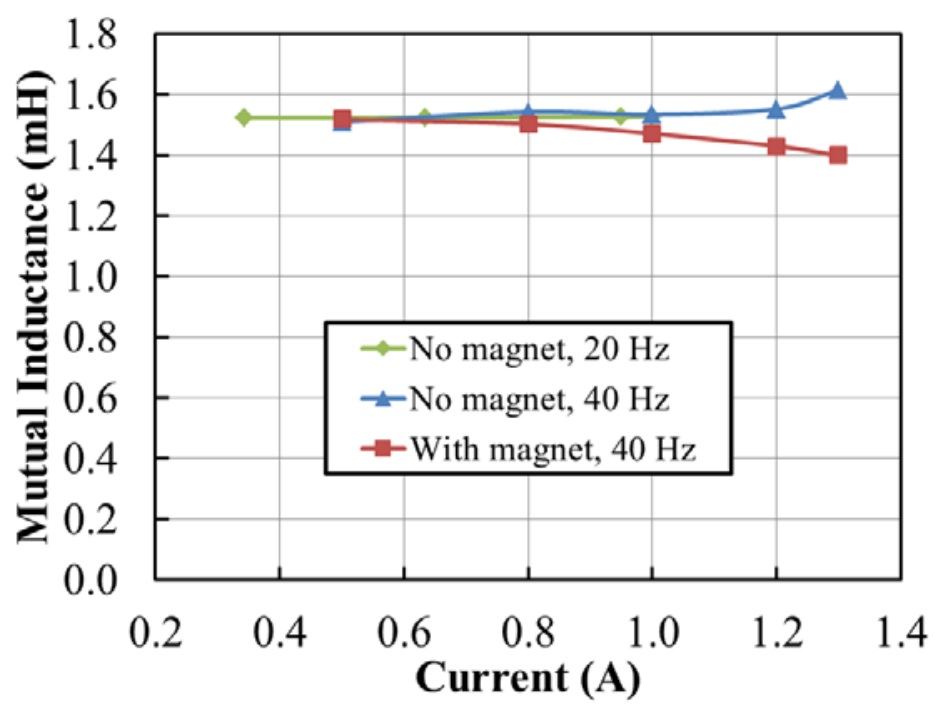

Fig. 6 Mutual inductances calculated from static tests

\subsection{Results and Discussion}

A series of tests on the linear compressor have been conducted at different strokes in vacuum. The piston displacement, drive current and search coil voltage were measured. Using the mutual inductance of $1.5 \mathrm{mH}$ from Section 3.3, the flux linkage can be calculated according to Equation (6). Fig. 7 shows the flux linkage variation with the piston displacement for a stroke of 11, 12 and $13 \mathrm{~mm}$. It can be seen that the three strokes give a very linear and similar relationship between the flux linkage and the displacement. According to Equation (8), piston stroke is expected to be a linear function of the peak-to-peak flux linkage $\Phi_{\max }-\Phi_{\min }$, and indeed Fig. 8 shows a very linear relation for a wide range of strokes, indicating that the piston stroke can be accurately detected by calculating the flux linkage in the search coil.

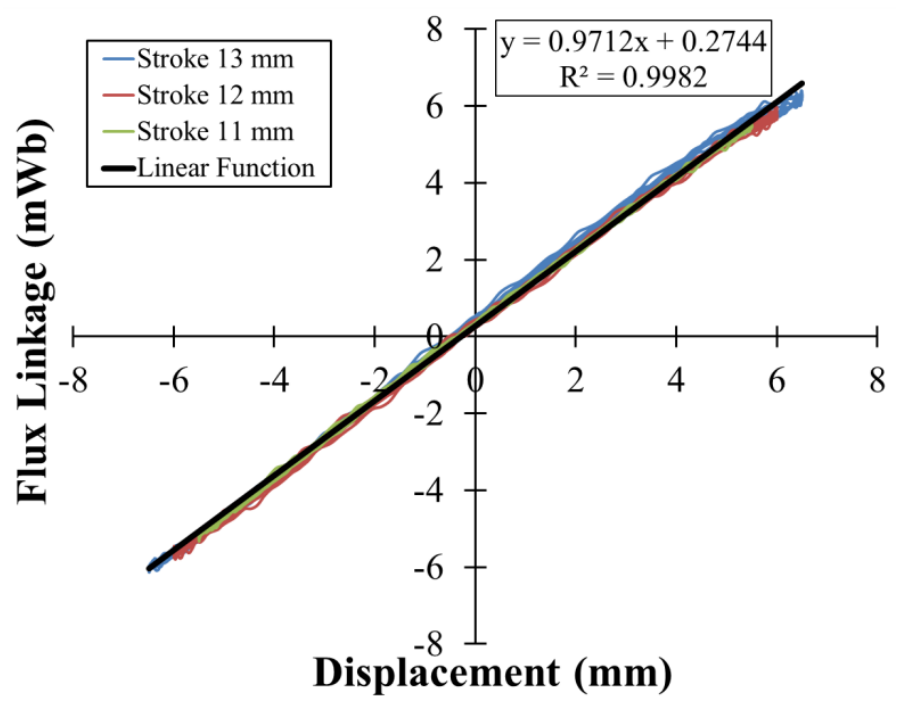

Fig. 7 Flux linkage variation with displacement at different strokes (13 mm, $12 \mathrm{~mm}$ and $11 \mathrm{~mm})$ with a mutual inductance of $1.5 \mathrm{mH}$ 


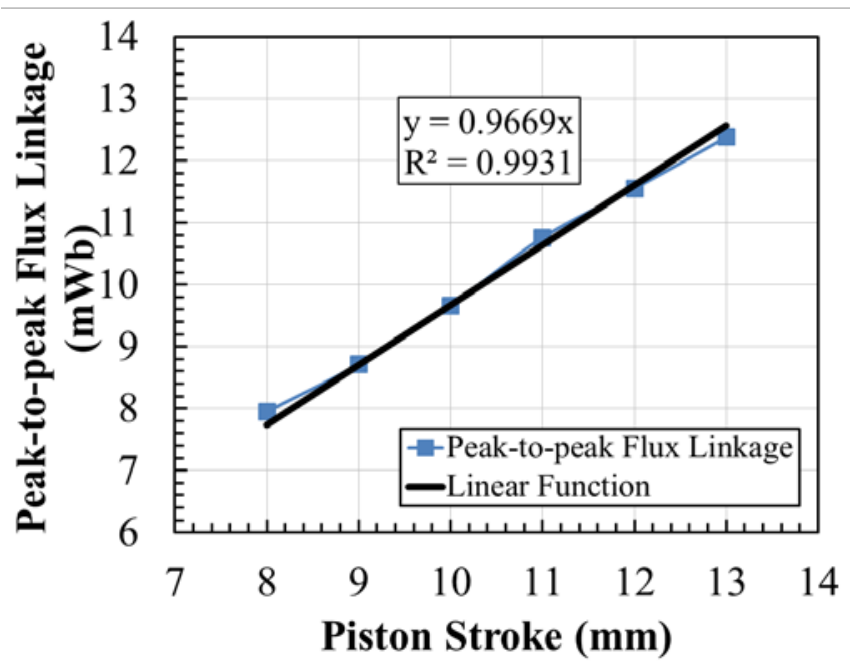

Fig. 8 Peak-to-peak flux linkage plotted against piston stroke with a mutual inductance of $1.5 \mathrm{mH}$ and a piston offset of zero

A series of tests have been undertaken with different piston offset at each stroke. The flux linkage difference $\Phi_{A}-\Phi_{B}$ was calculated according to Section 3.2. Fig. 9 shows the flux linkage difference as a function of the piston offset for various strokes with a mutual inductance of $1.5 \mathrm{mH}$. It can be seen that all five curves are very linear crossing at the same point close to the origin of the coordinates, although the slopes are slightly different from each other. When the piston offset is zero, the flux linkage difference is not zero, and this may be attributed to the accuracy of the reference pulse generation, and the measurements of displacement and current. To control the linear compressor, this value can be subtracted from the process variable of the controller.

A number of trials show that the relationship between the flux linkage difference and piston offset is not very sensitive to the mutual inductance within a range of 1.2-2.4 $\mathrm{mH}$. This is very useful because the piston offset can be linearly defined by the flux linkage difference that is calculated from the search coil voltage after subtracting the mutual inductance effect, and furthermore the mutual inductance does not need to be known very accurately. Previous static tests indicate a range of 1.3-1.6 $\mathrm{mH}$ for the mutual inductance, which is within the range where the results are insensitive to the value of the mutual inductance. 


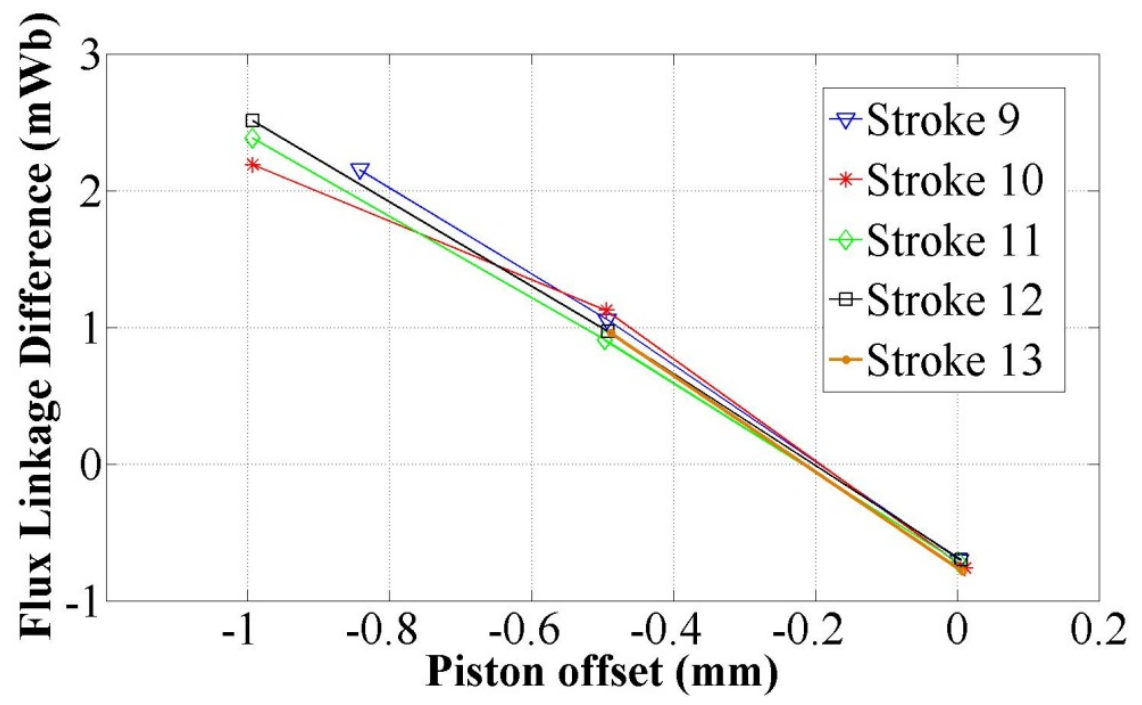

Fig. 9 Flux linkage difference plotted against piston offset for strokes of 9-13 mm with a mutual inductance $M$ of $1.5 \mathrm{mH}$

\section{Compressor Control}

Linear compressor control includes stroke control, offset control and frequency adjustment. Fig. 10 shows the schematic of the proposed compressor control system (the broken lines represent voltage signals from measurements into the control unit while the chain-dotted line represents the signal output from the control unit). The piston stroke control is achieved by measuring the current and voltage and varying the excitation (drive) voltage, with the piston stroke calculated according to Section 3.1. The piston offset is detected by using a search coil wound around the main coil with the inductive voltage measured by a voltage sensor, and a proximity sensor detecting when the piston passes the mechanical zero position (datum position). The control unit calculates the piston offset based on the flux linkage difference (see Section 3.2) and varies the duty cycle of the pulse on the solenoid valve to adjust the compressor body pressure to keep the piston centred. The drive frequency can be adjusted for resonant operation in response to different operating conditions so as to minimize the drive current and the ohmic losses. The proposed control system for the prototype linear compressor has been implemented by using the data acquisition and control modules in LabVIEW. A LVDT displacement transducer was employed to sense the actual piston position, to validate the control approach, and to generate a pulse when the piston passes the datum position (to represent the proximity sensor).

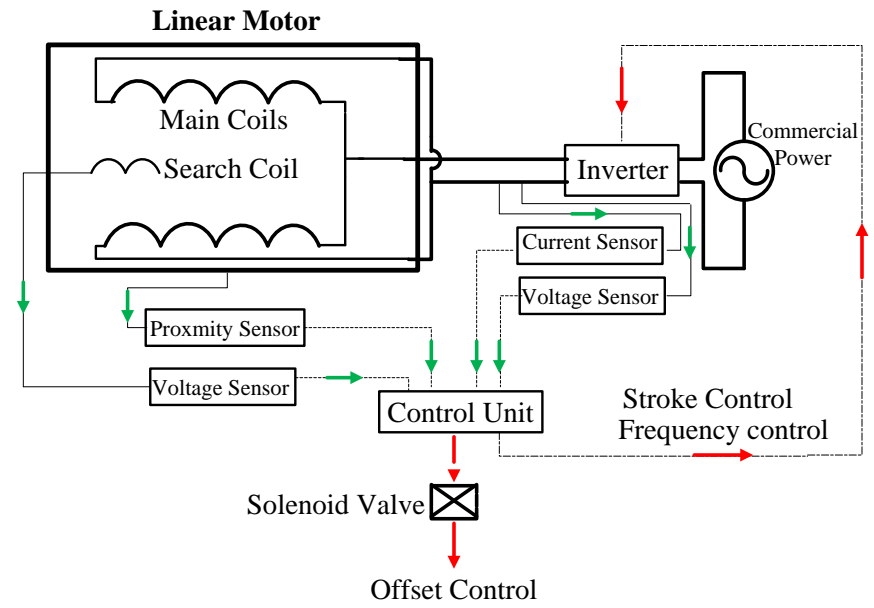


Fig. 10 Linear compressor control schematic

\subsection{Stroke Control}

Fig. 11 shows the block diagram of the PID controller for stroke control in the linear compressor. The set point is the stroke that the linear compressor should operate at in response to the load. The stroke is detected using a search coil and current and voltage sensors (see Section 3.1). The PID controller in LabVIEW calculates an 'error' value as the difference between a measured process variable (PV) and a desired set point (SP) for the stroke. The controller attempts to minimize the error by adjusting the process control output which is the amplitude of drive voltage waveform. A sinusoidal waveform is generated using an analogue output from the NI6251 data acquisition (DAQ) card. The operating frequency is set to resonance, as determined by the gas spring rate and the mechanical spring rate. The sinusoidal waveform goes into the power amplifier to provide electrical power input into the compressor. A capacitance box was employed to apply a power factor correction, as this reduces the voltage requirement for the amplifier. The amplitude of the waveform is adjusted to minimize the error between the set point and the instantaneous stroke calculated from the measured variables.

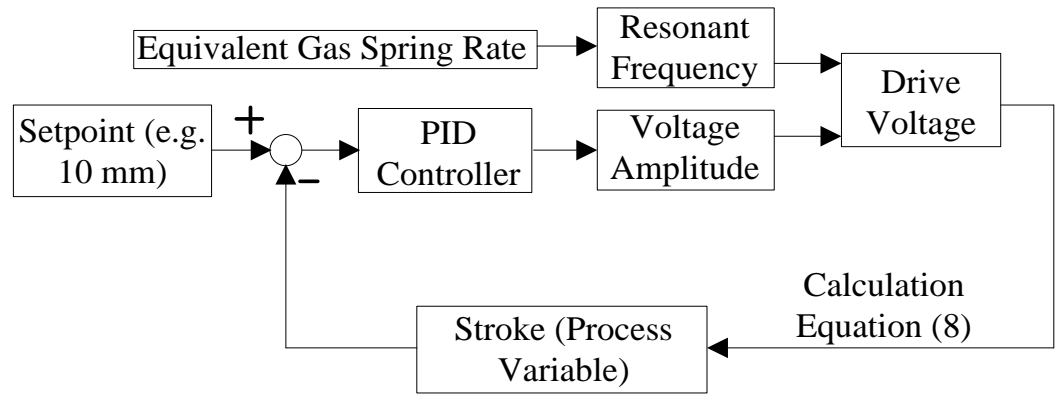

Fig. 11 Block diagram of the PID controller for stroke and frequency control of the linear compressor

An example of the stroke control with a set point of $10 \mathrm{~mm}$ using nitrogen as the working fluid is shown in Fig. 12. The amplitude for the siusoidal waveform was adjusted to bring the stroke from 0 to $10 \mathrm{~mm}$. Changing the operating frequency or pressure ratio will change the steady-state amplitude value for each target stroke. The piston stroke settled at $10 \mathrm{~mm}$ after 15 seconds from stationary state. Note that over-stroking occurred when reducing the frequency. Reducing the frequency by $0.5 \mathrm{~Hz}$ led to $0.2 \mathrm{~mm}$ over stroke, so the frequency should be changed slowly for higher stroke operation.

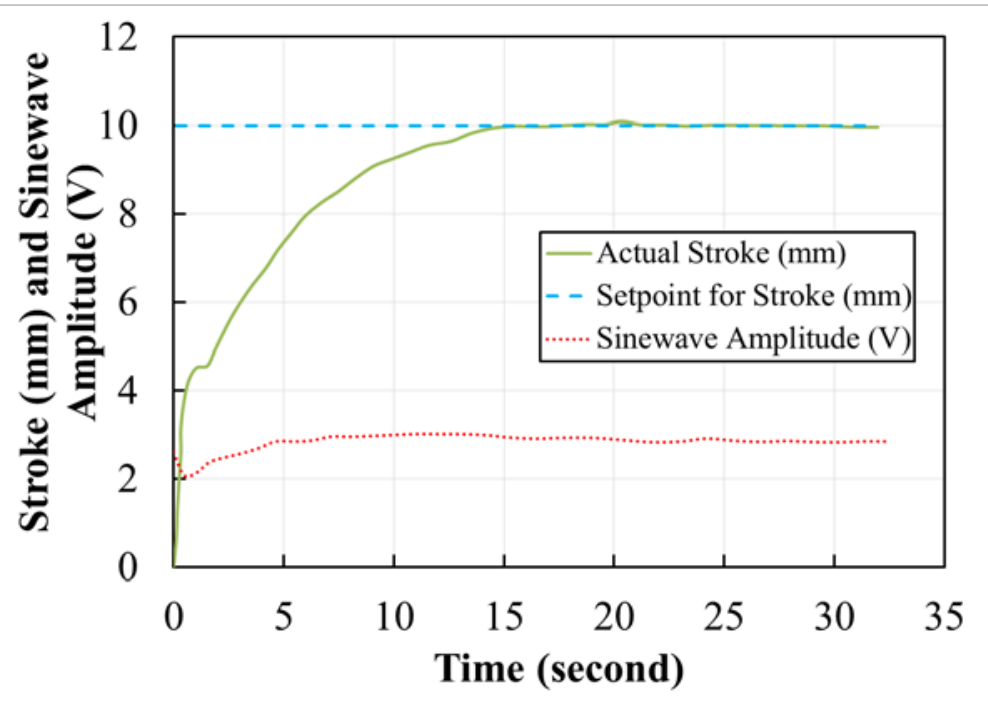


Fig. 12 Stroke variations in response to the PID controller with a set point step change from 0 to 10 $\mathrm{mm}$

\subsection{Offset Control}

A bleed flow has been used to vary the body side pressure $\left(P_{\mathrm{b}}\right.$ in Equation 1$)$ to control the offset. A PID controller has been developed in LabVIEW for the offset control. The process variable is the flux linkage difference calculated by using the search coil (see Section 3.2). The set point for the offset is the desired value of $0 \mathrm{~mm}$ which means that the piston oscillates about the 'mechanical zero' of the springs. The PID controller determines the duty cycle of the pulse applied to the solenoid valve, which drives the offset towards the set point by changing the bleed flow rate to reduce the body pressure. The digital pulse train that makes up the PWM (pulse-width-modulation) signal has a fixed frequency and varies the pulse width to alter the average power of the signal controlling the analogue circuit. The block diagram of the PID controller for the offset is illustrated in Fig. 13.

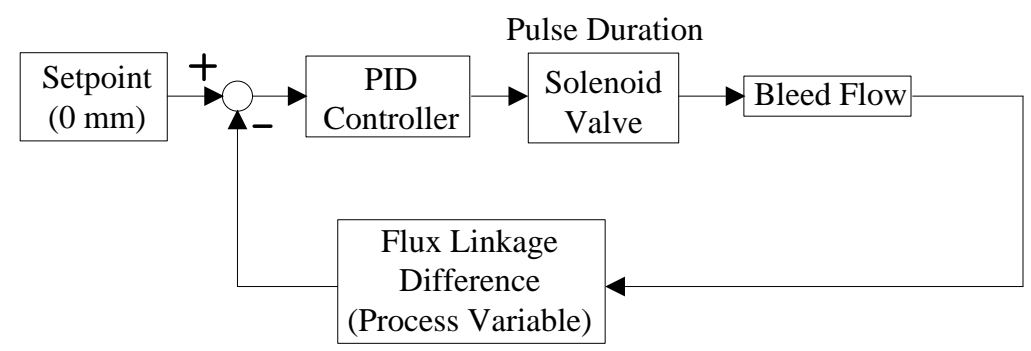

Fig. 13 Block diagram of the PID controller for the stroke and frequency control in the linear compressor

An example of the offset control with a set point of 0 using nitrogen as working fluid is shown in Fig. 14, when the linear compressor was operated at a stroke of $10 \mathrm{~mm}$ and had an initial offset of $-1.0 \mathrm{~mm}$ (note that negative displacement means towards the cylinder head from the datum position). A series of pulses with a frequency of $40 \mathrm{~Hz}$ and changing duty cycle were generated to reduce the offset to 0 $\mathrm{mm}$ after 50 seconds. 


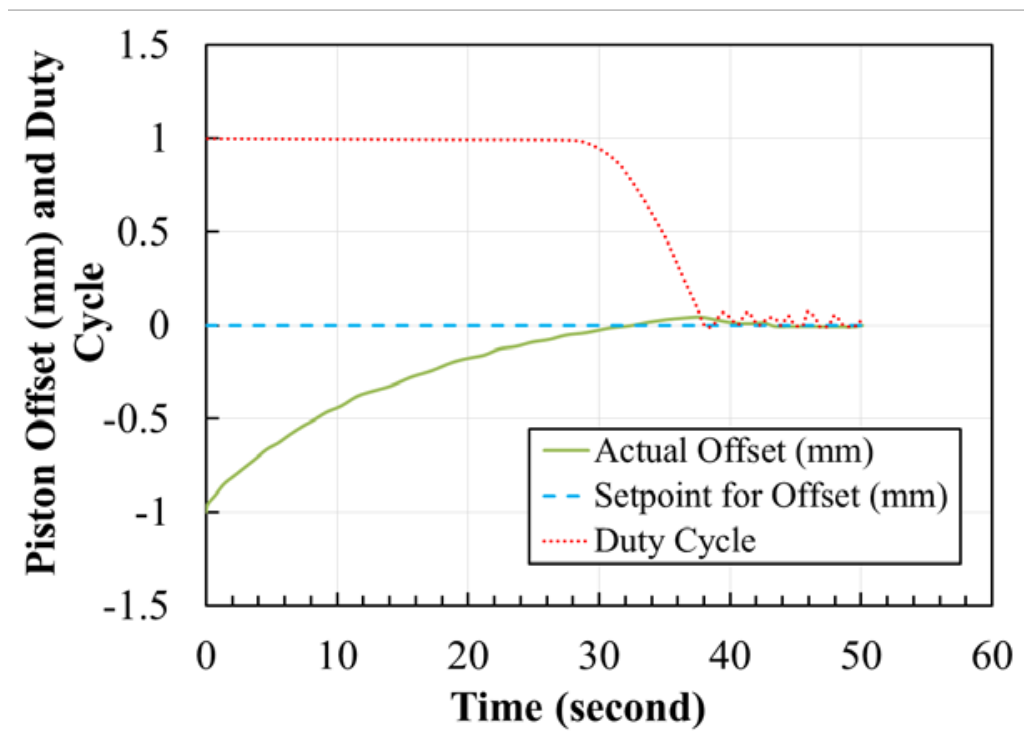

Fig. 14 Offset variations in response to the PID controller using solenoid valve (stroke of $10 \mathrm{~mm}$ )

A series of control system tests have been conducted by changing the PWM frequency from 1 to 40 $\mathrm{Hz}$ for a test condition of a pressure ratio of 4 and a stroke of $10 \mathrm{~mm}$. The settling time remains same for different PWM frequencies when the PID gains are fixed. The only difference for the different operating frequencies is the duty cycle at steady-state which varies from $4.4 \%$ at $10 \mathrm{~Hz}$ to $6.8 \%$ at 1 $\mathrm{Hz}$. Therefore, the solenoid valve for the offset control can operate at a frequency of $1 \mathrm{~Hz}$, and this should the durability.

\subsection{Resonant Frequency}

As has been reported by Pollak et al. (1979), the linearized gas spring force was divided by the stroke of the compressor, to yield an estimate of the effective spring rate of the gas in the compression chamber:

$$
k_{\mathrm{g}}=\frac{\left(P_{\mathrm{dis}}-P_{\mathrm{suc}}\right) A}{S}
$$

Therefore, the total stiffness (including the effective mechanical spring stiffness, $k_{\mathrm{m}}$ from Table 1 ) is

$$
k=k_{\mathrm{g}}+k_{\mathrm{m}}
$$

The resonant frequency $f$ (in $\mathrm{Hz}$ ) can, therefore, be calculated as

$$
f=\frac{1}{2 \pi} \sqrt{\frac{k}{m}}
$$

where $m$ is the moving mass (magnet and piston assembly) given in Table 1.

Fig. 15 shows a comparison between the measurements and calculations of the resonant frequency for different stroke points at each pressure ratio from the nitrogen tests. The maximum error is only about $3 \%$ and the average error is about $1.8 \%$. From this, it can be concluded that the resonant frequency can be accurately modelled based on: the discharge pressure, the suction pressure and the stroke. The suction and discharge pressure correspond with condenser and evaporator temperatures. Though this approach is an approximation, it is generally accurate enough and has the advantage of simplicity. 
The assumption of a linear gas spring is acceptable for low pressure ratios. However, when the linear compressor is operated at high pressure ratios (e.g. above 4), the gas spring becomes significantly non-linear. The effective gas spring rate then needs to be modelled in more detail so as to retain sufficient accuracy.

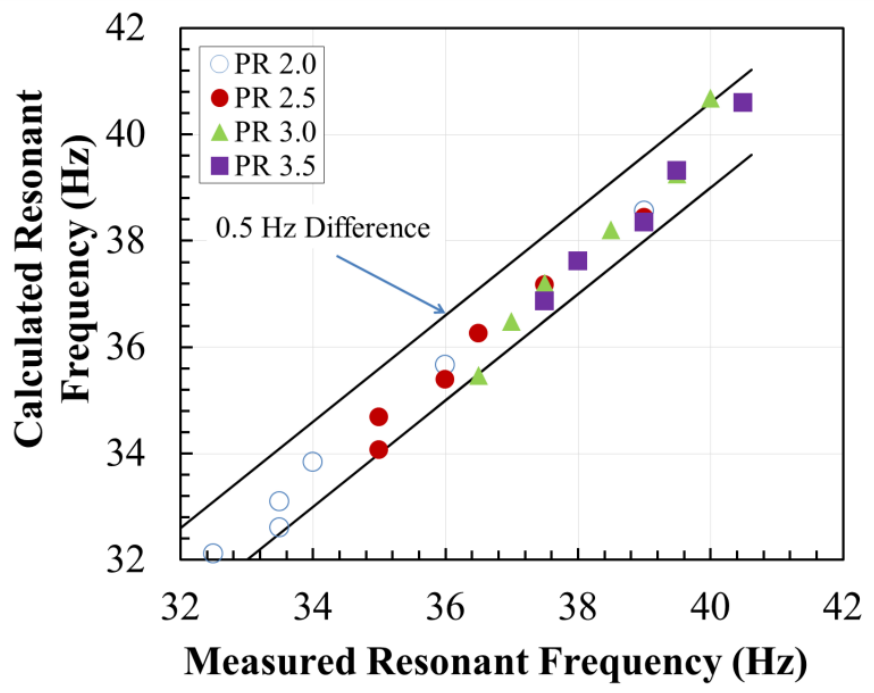

Fig. 15 Comparison between the calculated and measured resonant frequency at different pressure ratios from the linear compressor tests using nitrogen

\subsection{Experimental Uncertainty}

A number of parameters were measured in the experiments, including pressures, stroke, frequency, current, voltage and electrical power. Typically, a set of readings was taken every 20 minutes so as to allow time for thermal equilibrium to be attained. The measurements of pressure, stroke and electrical power have absolute uncertainties of 0.015 bar, $0.02 \mathrm{~mm}$ and $5 \mathrm{~mW}$ respectively. The current and voltage transducers have accuracies of $0.5 \%$ and $0.25 \%$ respectively. The combined uncertainties of the calculated values were calculated using a 95\% confidence interval. The flux linkage, mutual inductance, and frequency have absolute uncertainty values of $0.012 \mathrm{mWb}, 0.008 \mathrm{mH}$, and $0.003 \mathrm{~Hz}$, respectively.

\section{Conclusions}

An approach to detecting the piston position of the linear compressor has been described using a search coil for both stroke and offset control. The offset control used a solenoid valve to control the bleed flow rate. The operating frequency for resonance can be modelled and controlled. The proposed control technique does not require a position transducer but a proximity sensor that detects the datum position. Control of the frequency, displacement and piston offset can be easily implemented in a digital controller. Experimental results validate the proposed compressor control technique.

(1) The piston stroke is a linear function of the peak-to-peak flux linkage in the search coil when the offset is zero. The peak-to-peak flux linkage calculation does not require integration constant. 
(2) The piston offset is a linear function of a flux linkage difference in the search coil defined as described in Section 3.2. The calculated flux linkage difference can be used as the process variable to drive a controller. The advantage with this approach is that the target value is zero and an accurate relationship between position and flux linkage is not required.

(3) Both piston stroke and offset detections require an input value of mutual inductance between the search coil and the main coil. Experiments show an average mutual inductance of $1.5 \mathrm{mH}$, but this does not need to be known accurately.

(4) The piston stroke is controlled by adjusting the amplitude of the sinusoidal waveform of the voltage which varies with pressures and frequency. The settling time for the stroke is $15 \mathrm{~s}$ from a stationary state to a $10 \mathrm{~mm}$ stroke.

(5) The piston offset is achieved by adding a bleed flow from the compressor body to the suction line of the compressor. The bleed flow rate is controlled by a solenoid valve with pulse width modulation by a PID controller. The pulse can be generated at $1 \mathrm{~Hz}$ indicating a simple, durable and cheap solenoid valve that is suitable for mass production.

(6) The resonant frequency for each operating condition (in terms of suction and delivery pressure and stroke) can be estimated. Experiments show that the maximum error is less than $1 \mathrm{~Hz}$.

(7) The proposed compressor control system has been successfully implemented in LabVIEW indicating a feasible and effective solution for large scale manufacture.

\section{Acknowledgement}

The authors would like to thank the EPSRC for their financial support throughout the construction of the linear compressor (Grant Ref EP/E036899/1).

\section{References}

Bailey, P.B., Dadd, M.W., and Stone, C.R., 2010-2011. Cool and straight: linear compressors for refrigeration. In: Proc. Inst. Refrigeration, 4-1.

Bradshaw, C.R., Groll, E.A., and Garimella, S.V., 2011. A comprehensive model of miniature-scale linear compressor for electronics cooling. Int. J. Refrigeration, 34, pp. 63-73.

Many Sunpower papers are available at http://www.sunpower.com/library/coolingcompression.php. http://www.lg.com/uk/linear/index.

http://www.acr-news.com/news/news.asp?id=2231.

http://www.embraco.com/wisemotion/Default.aspx.

Bradshaw, C.R., 2012. A miniature-scale linear compressor for electronics cooling. Ph.D. Thesis, Purdue University.

Tassou, S.A., Marquand, C.J., and Wilson, D.R., 1983. Comparison of the performance of capacity controlled and conventional on/off controlled heat pumps. Applied Energy, 14, pp. 241-256.

Huang, B.J. and Chen, Y.C., 2002. System dynamics and control of a linear compressor for stroke and frequency adjustment. J. Dynamic Systems, Measurement, and Control, 124, pp. 176-182.

Kim, J.K. and Jeong, J.H., 2013. Performance characteristics of a capacity modulated linear compressor for home refrigerators. Int. J. Refrigeration, 36, pp. 776-785.

Kim, J.K., Roh, C.G., Kim H., and Jeong, J.H., 2011. An experimental and numerical study on an inherent capacity modulated linear compressor for home refrigerators. Int. J. Refrigeration, 34, pp. 1415-1423.

Kim, H., Roh, C.K., Kim, J.K., 2009. An experimental and numerical study on dynamic characteristic of linear compressor in refrigeration system. Int. J. Refrigeration, 32, pp. 1536-1543. 
Spoor, P.S., and Corey, J.A., 2004. A novel method for controlling piston drift in devices with clearance seals. Cryocoolers, 13, pp. 215-223.

Zou, H.-M., Shao, S.-Q., Tang, M.-S., Zhang, L.-Q., and Peng, G.-H., 2012. Performance investigation of linear compressor with one side springs. Proc. Intl. Compressor Engineering Conf., Purdue, 1115, pp. 1-7.

Choe, G., and Kim, K., 2000. Analysis of nonlinear dynamics in a linear compressor. Int. J. Series C Japan Society of Mechanical Engineers, 43(3), pp. 545-552.

Liang, K., Stone, C.R., Dadd, M.W., and Bailey, P.B., 2014. A novel linear electromagnetic-drive oilfree refrigeration compressor using R134a. Int. J. Refrigeration, 40, pp. 450-459.

Liang, K., Dadd, M.W., and Bailey, P.B., 2012. Clearance seal compressors with linear motor drives part i: background and system analysis. Proc. Inst. Mechanical Engineering, Part A: J. Power and Energy, 227, No. 3, pp. 252-260.

Bailey, P.B., Dadd, M.W., and Stone, C.R., 2009. An oil-free linear compressor for use with compact heat exchangers. Proc. Intl. Conf. on Compressors and their Systems, IMechE, London, pp. 259268.

Pollak, E., Soedel, W., Cohen, R., and Friedlaender, F.J., 1979. On the resonance and operational behavior of an sscillating electrodynamic compressor. J. Sound and Vibration, 67(1), pp. 121-133. 